\title{
A Precise Electrical Disturbance Generator for Neural Network Training with Real Level Output
}

\author{
Antonio García, Carlos León, Iñigo Monedero, \\ and Jorge Ropero \\ Escuela Técnica Superior de Ingeniería Informática \\ Departamento de Tecnología Electrónica \\ Avda, Reina Mercedes s/n \\ 41012 Sevilla (Spain) \\ imonedero@us.es
}

\begin{abstract}
Power Quality is defined as the study of the quality of electric power lines. The detection and classification of the different disturbances which cause power quality problems is a difficult task which requires a high level of engineering expertise. Thus, neural networks are usually a good choice for the detection and classification of these disturbances. This paper describes a powerful tool, developed by the Institute for Natural Resources and Agrobiology at the Scientific Research Council (CSIC) and the Electronic Technology Department at the University of Seville, which generates electrical patterns of disturbances for the training of neural networks for PQ tasks. This system has been expanded to other applications (as comparative test between PQ meters, or test of effects of power-line disturbances on equipment) through the addition of a specifically developed high fidelity power amplifier, which allows the generation of disturbed signals at real levels.
\end{abstract}

Keywords: Power quality, electrical disturbance, neural network.

\section{Introduction}

Power Quality (PQ) is defined as the study of the quality of electric power lines. PQ has been a topic of consideration for the last two decades, and has recently acquired intensified interest due to the wide spread use of electronic devices in complicated industrial processes and the generalized power quality of commercial electric power [1]. Thus nowadays, customers demand higher levels of PQ to ensure the proper and continued operation of such sensitive equipment.

The poor quality of electrical power is normally attributed to power line disturbances such as waveshape faults, overvoltages, capacitor switching transients, harmonic distortion and impulse transients. Thus, electromagnetic transients, which are momentary voltage surges powerful enough to shatter a generator shaft, can cause catastrophic damage suddenly. Harmonics, sometimes referred to as electrical pollution, are distortions of the normal voltage waveforms found in ac transmission, which can arise at virtually any point in a power system. While harmonics can be as destructive as transients, often the greatest damage from these distortions lies in the loss of 
credibility of the power utilities vis-a-vis their customers. The classification and identification of each one of the disturbances is normally carried out from standards and recommendations depending on where the utilities operate (IEEE in the United States, UNE in Spain, etc).

The detection and classification of the different disturbances which cause power quality problems is a difficult task which requires a high level of engineering expertise [2]. Due to the above mentioned difficulties, artificial intelligence tools [3] emerge as an interesting alternative in the detection of electrical disturbances. The main intelligent tools of interest include expert systems, fuzzy logic and artificial neural networks (ANNs) [4].

\section{Neural Networks on Power Quality}

For the detection and classification of disturbances, ANNs can be combined with mathematical analysis such as Fourier and Wavelet transforms for the generation of signal features which serve as inputs in the network [5]. Thus, feature extraction by wavelet transforms provides an unique characteristic which can represent every single PQ disturbance at different resolutions using the technique called multi-resolution signal decomposition or multi resolution analysis. In this way, while the detection of the power quality signals has tended to be easy, their classification is still a difficult task in which ANNs play an important role [6][7][8].

Pattern recognition in ANNs generally requires preprocessing of data, feature extraction and final classification. One of the most important tasks in the design and development process of an ANN is to generate an adequate number of training patterns in order to approximate future inputs. Sometimes an optimal design of the ANN is found but the limited number of train patterns does not give good results. In particular, in PQ a great numbers of electrical patterns are necessary due to the multiple combinations of different disturbances which can coincide in one or various samples. Another additional problem with ANNs applied to PQ is the impossibility of getting real patterns directly from the power line due to the irregularity in the apparition of disturbances.

\section{Electrical Disturbance Generator}

\subsection{General Description}

Initially the function of this system was to allow the generation of training signals at level of files of data. Nevertheless, as we described next, has added to an interface (D/A converter and power amplifier) that allows the generation of these signals at the levels of tension of power grid with medium power levels.

This Generator of disturbances, therefore, it has been developed in two different stages: the generator of electrical patterns and the power amplifier:

In one first stage the generating subsystem of patterns has been developed (named with the acronym PANDORA). This system allows the programmable generation of signals that emulate the operation of the power grid, allowing the easy addition of disturbances on it. 


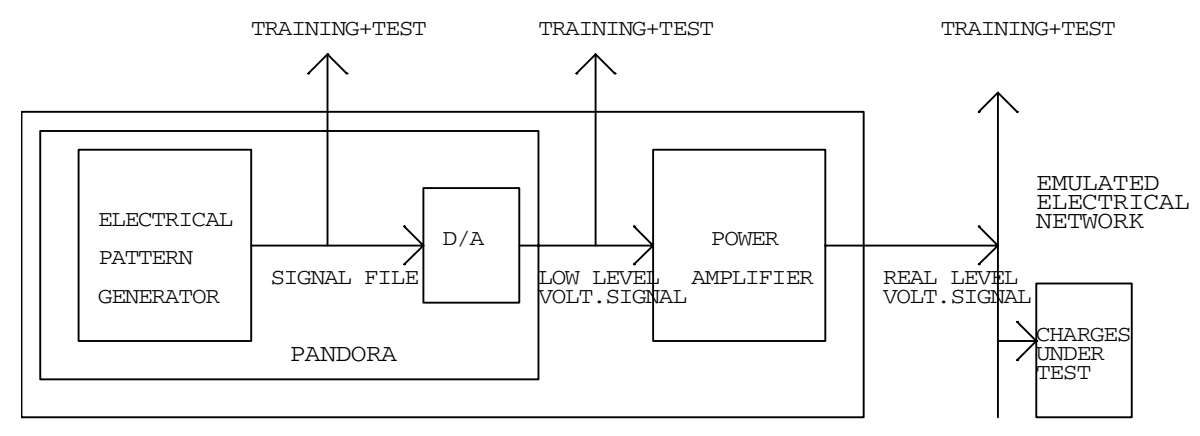

ELECTRICAL DISTURBANCE GENERATOR

Fig. 1. Electrical Disturbance Generator. General Scheme.

The PANDORA allows to obtain the signals at level of files (sequence of numeric data) and by means of the use of a D/A board of signals of tension of low level (tensions between +/- 10 volts. and powers of milliwatts). These two forms of outputs have been used to train the ANN of the system detector of disturbances, and later to check its operation.

The second stage has corresponded to the design, implementation, and test of the power amplifier. The function of this amplifier is to elevate the level of the signals coming from the PANDORA, until real existing in the network (the signals of the order of 230 volts rms and values of power of the order of at least hundreds of watts).

The amplifying system, in combination with subsystem PANDORA, completes the functionality of the generating system of disturbances allowing:

1. The systematic test at levels of real power grid of our detector of disturbances in a reasonable term. Until the development of the amplifying system, the single detector of disturbances could be made a will of complete form by means of its connection to real power grid. The tests made in this sense have been quite satisfactory, and the detector has worked correctly before the disturbances that have taken place. Nevertheless, considering, that real power grid has relatively high quality, this is, that the disturbances that take place are, in general, little frequent, and in addition unforeseeable, the produced disturbances are few for an extensive time of operation

2. The comparison of our detector of disturbances with other similar equipment of the market. This is an essential task as opposed to demonstrate the advantages of our system of detection of disturbances to the alternative techniques. With the Electrical Disturbance Generator is possible to establish systematic protocols of comparative tests between equipment based on different techniques and to verify the limits and precision of the different techniques.

3. Test of equipment under disturbances of the network [10][11]. The possibility of having a system able to generate signals equivalent to those of real power grid, with the possibility of generating of arbitrary form disturbances in the same one it allows a fan of very interesting possibilities: 
* It allows the manufacturers of equipment to have a tool to the test of its equipment before the disturbances that can take place in power grid, allowing to the verification of its immunity or its improvement before this type of effects.

* It allows the providing companies to make defensive analyses before claims of the users by failures of equipment, demonstrating, for example, that this equipment fails before disturbances that do not leave the limits established by the norm.

\subsection{Programmable Electrical Pattern Generator (PANDORA)}

For the task of training neural networks for the detection and classification of electrical disturbances we are developing an electrical pattern generator. The objective of this generator is to create an unlimited number of patterns to be used by a classification system.

In particular, we are developing a classification system in the Electronic Technology Department at the University of Seville and the Institute for Natural Resources and Agrobiology. The system is a real-time detector of power line disturbances based on artificial intelligence techniques (in particular, a first version based on ANNs) is being developed. We are going to use the Electrical Pattern Generator in order to carry out the ANN training.

The Electrical Pattern Generator make it possible to configure parameters such as the duration of the sample, the frequency of the signal and the number of samples in an ideal cycle $(50 \mathrm{~Hz}$ or $60 \mathrm{~Hz})$ and to add one or more disturbances. From the selected parameters, the generator creates a text file with the voltage values of the sample. The structure of the file consists of a header with the file information (name, number of sample cycles and sampling period) and a data column corresponding to the voltages of each of the samples.

The type of disturbances includes: impulse, oscillation, sag, swell, interruption, undervoltage, overvoltage, harmonics, flicker and frequency variations. In amplitude disturbances (impulses, sags, swells, interruptions, undervoltages and overvoltages), the tool allows us that parameters such as amplitude, start time, final time, rising and falling slope, be configured. The edition of harmonics allows the configuration of amplitude and phases as far as forty harmonic levels including the possibility of adding them an offset. In the flicker menu the start time can be set and the final time of the flicker, its RMS, frequency and phase.

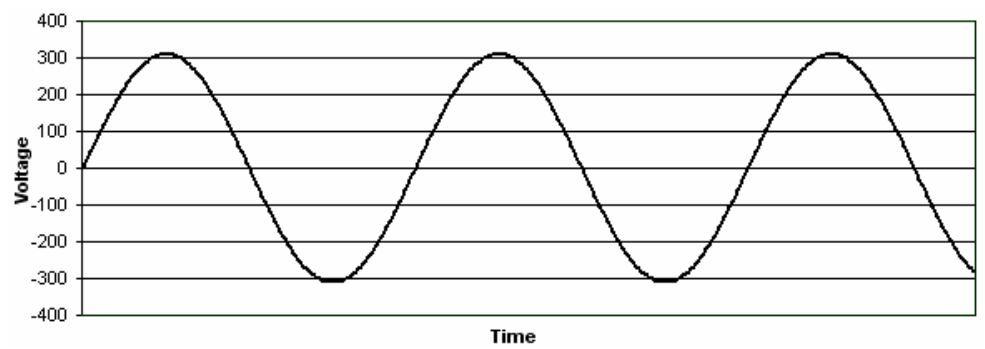

Fig. 2. Ideal signal (one-phase representation) 


\section{Models of disturbances}

1.- Harmonic distortion

Harmonic distortion is defined as the phenomenon in which diverse sinusoidal signals with diverse frequencies which are multiples of the fundamental frequency are superposed on the ideal signal (Figure 3).

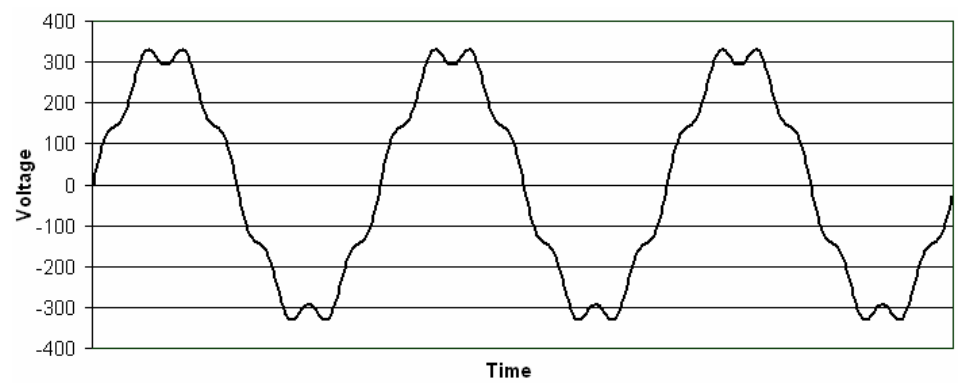

Fig. 3. Harmonic distortion

The following mathematical model was implemented in the generator:

$$
C(t)=A+\sum_{i=1}^{N} A_{i} \sin \left(2 \pi f_{i} t+\varphi_{i}\right)
$$

- $A$ : DC term $(\mathrm{V})$.

- $A_{i}$ : Amplitude of the $i$ th harmonic of signal (V).

- $f_{i}$ : Frequency of the $i$ th harmonic of signal $(\mathrm{Hz})$

- $\varphi_{i}:$ Phase of the ith harmonic (Rad)

- $i$ : Harmonic order $(i=1, \ldots, N)$.

In our harmonic model $C(t)$ is considered as consisting of a fundamental and 39 harmonic components.

2.- Frequency deviation

Frequency deviation is a signal disturbance added to the harmonic distortion. The model consists of the frequency modulation of the signal $C(t)$ by means of the carrier signal $M(t)$, which is named modulating signal.

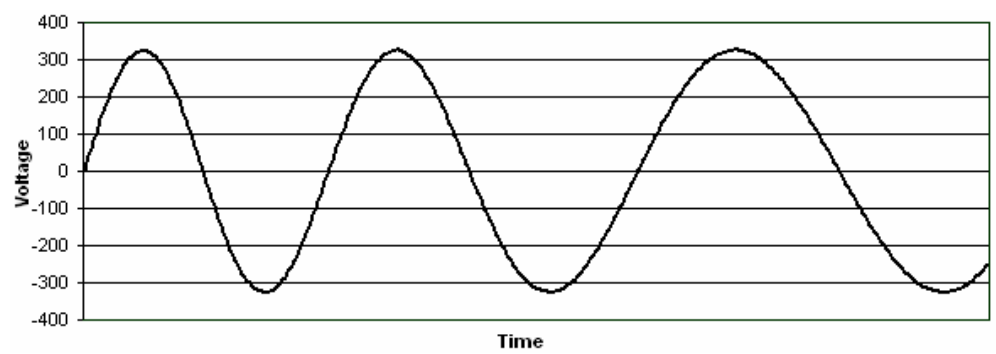

Fig. 4. Frequency deviation 
The mathematical expression of this signal is:

$$
M(t)=B+\sum_{j=1}^{40} B_{j} \sin \left(2 \pi j f_{m} t+\varphi_{j}\right)
$$

- $\quad B$ : DC term (V).

- $\quad B_{j}$ : Amplitude of the $j$ th harmonic of signal (V)

- $f_{m}$ : Fundamental frequency $(\mathrm{Hz})$

- $\varphi_{i}$ : Phase of the $i$ th harmonic (Rad)

Considering (1) and (2) the resultant signal would end up as:

$$
X(t)=A+\sum_{i=1}^{40} A_{i} \sin \left[2 \pi i f_{c} t+\varphi_{i}+B+\sum_{j=1}^{40} B_{j} \sin \left(2 \pi j f_{m} t+\varphi_{j}\right)\right]
$$

which shows the harmonic content and frequency modulation.

The addition of another kind of disturbance was carried out from the previous expression $\mathrm{X}(\mathrm{t})$. Therefore, the previous expression would be the result of an ideal electrical signal or a frequency or/and harmonic disturbed signal.

\section{3.- Flicker}

Flicker (Figure 5) is considered an amplitude modulation of the carried signal $\mathrm{X}(\mathrm{t})$, which changes in function of the modulating signal $F(t)$ [9]. The modulating signal has sinusoidal form with prefixed random amplitude, frequency (usually around 30 $\mathrm{Hz}$ ) and an initial phase.

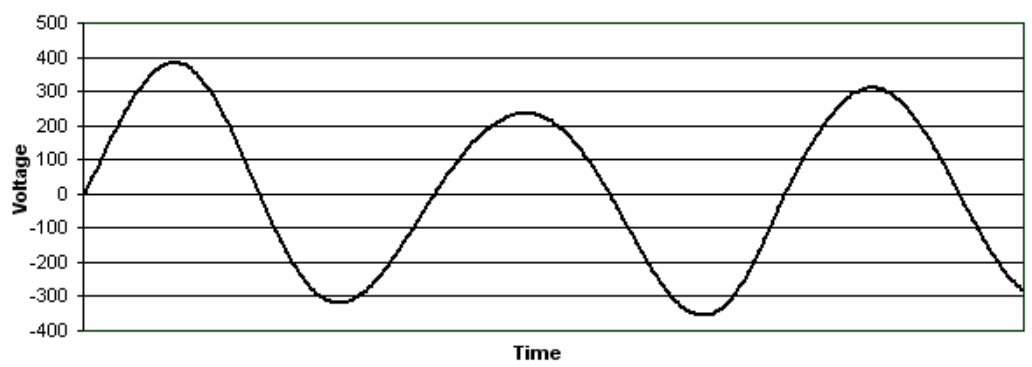

Fig. 5. Flicker

The equation which defines the mathematical model implemented by the pattern generator is:

$$
Z(t)=F(t) X(t)=\left[1+A_{f k} \sin \left(2 \pi f_{f k} t+\varphi_{f k}\right)\right] X(t)
$$

- $\quad A_{f k}$ : flicker amplitude

- $f_{f k}$ : flicker frequency

- $\varphi_{f k}$ : flicker phase

4.- Overvoltages, swells, undervoltages and sags.

In this kind of disturbances the amplitude of the signal rises (overvoltages or swells) or falls (undervoltages and sags) a certain value along a time interval. 


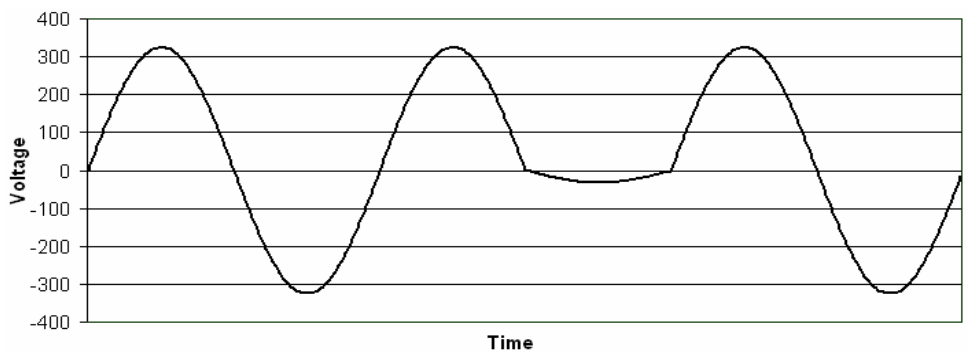

Fig. 6. Sag

In the development of the disturbance generator, a trapezoidal model for the amplitude evolution (lineal slope) was considered. The model makes it possible to approximate the amplitude disturbances most frequently encountered in power systems. Figure 7 shows a graphical of the model used for overvoltages or swells (inverse trapeze for undervoltages and sags).

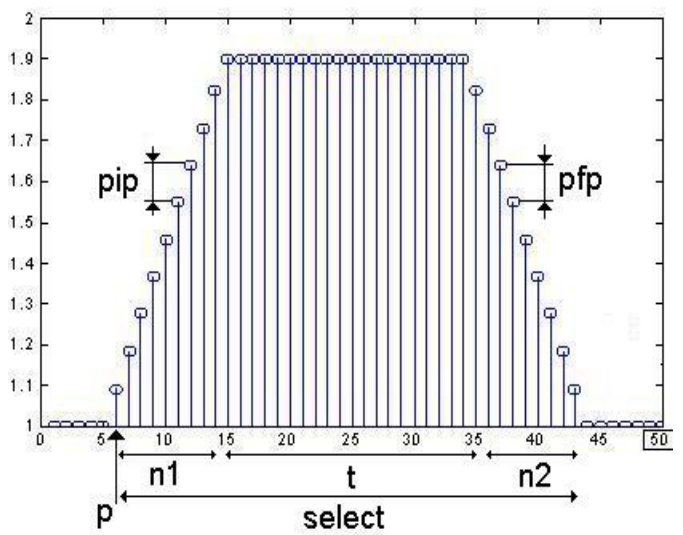

Fig. 7. Overvoltage and swell model

- $\quad p$ : initial sample of the trapeze

- $\quad$ pip: slope of the initial ramp

- pfp: slope of the final ramp

- $\mathrm{n} 1$ : number of samples of the initial ramp

- $\quad$ 2: number of samples of the final ramp

- $t$ : number of samples when the climb is reached

- select: total number of samples

5.- Transients

The electrical pattern generator models transients as a damped sine through a superposed exponential function, which is added to $X(t)$ at a certain point. 


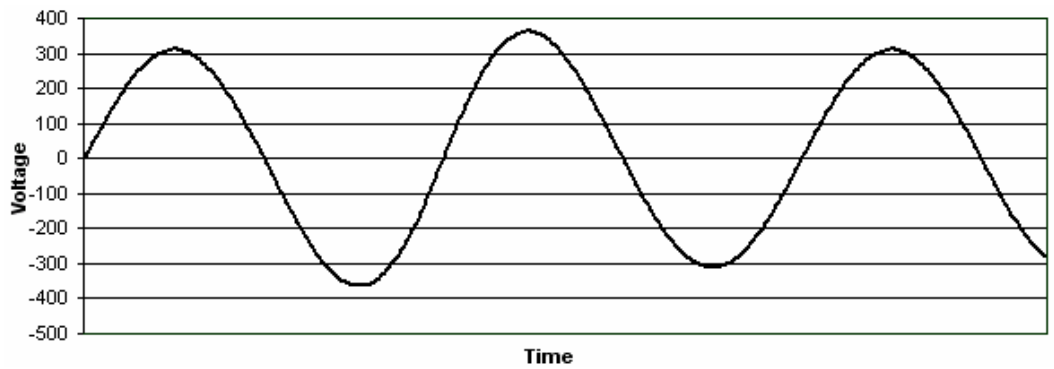

Fig. 8. Overvoltage

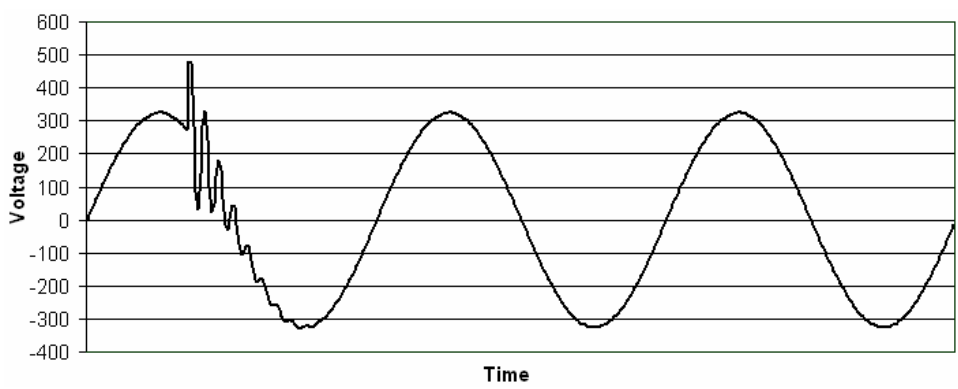

Fig. 9. Transient

The implemented mathematical model obeys to:

$$
T(t)=e^{-a t} A_{r} \sin \left(2 \pi f_{r} t+\varphi_{r}\right)
$$

- $\quad a$ : transitory exponent.

- $A_{r}$ : amplitude of the ripple (V)

- $f_{r}$ : frequency of the ripple $(\mathrm{Hz})$

- $\varphi_{r}$ : initial phase of the ripple (Rad)

6.- Noise

The generator makes it possible to add Additive White Gaussian Noise (AWGN) (Figure 10) in order to simulate more realistic signals of the power line.

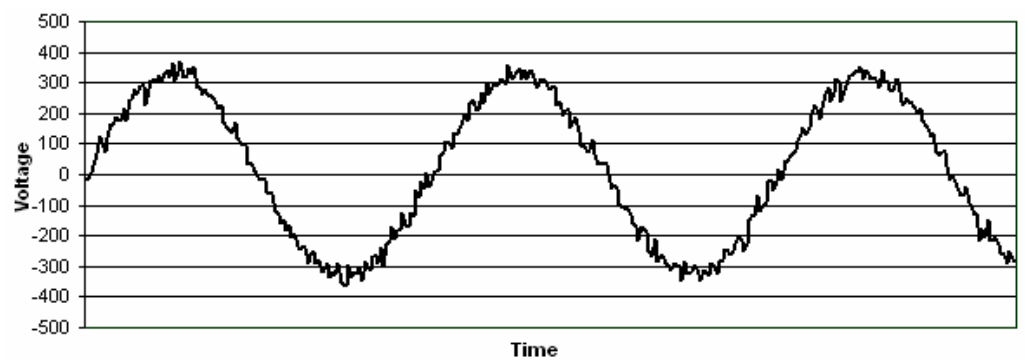

Fig. 10. Additive White Gaussian Noise (AWGN) 


\subsection{The Power Amplifier}

The power amplifier was specifically developed for the application. The main requirements were:

- $\quad$ Output Voltage up to: +/- 600 volts (instantaneous), 400 (RMS)

- $\quad$ Power, up to 200 watts.

- $\quad$ Band Width (minimum) ( $25 \mathrm{~Hz}$ to $5 \mathrm{Khz}$ )

- $\quad$ High fidelity. That implies: THD $<0.2 \%$, and $\mathrm{SNR}>60 \mathrm{~dB}$.

\section{Architecture}

This amplifier consists of:

- Module 1.- Electronic Voltage Amplifier. Class AB on C.I. (LM3886 of National Sem.). It will amplify the signals at medium levels of tension (20 40 volts rms) with medium powers ( $>100$ watts).

- Module 2.- Coupler Transformer. It will elevate the voltage (trafo ratio 1:10) at the required levels of tension. It will establish an input/output galvanic isolation.

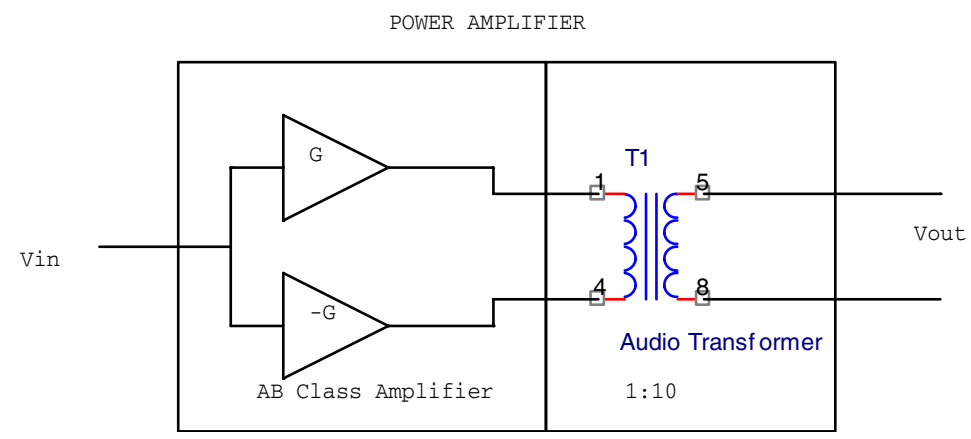

Fig. 11. Proposed Amplifier Architecture

The use of a transformer like element of connection to the load isolates the input and the output and allows to work with amplifiers at low voltage level. A medium power (500watts) audio professional transformer has been chosen to avoid the problems of bandwidth limitation and distortion that implies another type of transformers.

For the electronic amplifier the chosen configuration has been type $\mathrm{AB}$ as opposed to other types (example: D Type), due to its smaller distortion. On the other hand, the levels of power output are not as elevated as that the energy efficiency was the key question. It has been decided on a design based on Power Linear I.C. (LM3886 of National Sem.) to simplify the design because the device includes all the protection subsystems.

\section{$\underline{\text { System Tests }}$}

The system was put under a complete set of typical tests, and the obtained general results have been satisfactory in all the parameters: 
Table 1. Test results

\begin{tabular}{|l|l|}
\hline \multicolumn{2}{|c|}{ Characteristics } \\
\hline 1.-Input Impedance & $>25 \mathrm{Koh}$ \\
\hline 2.- Output Imp. & $<10 \mathrm{Oh}$ \\
\hline 5.- Gain & Up to 450 \\
\hline 6.- Band width & $<10 \mathrm{~Hz} />20 \mathrm{Khz}$ \\
\hline 7.- Vout(max) & $420(\mathrm{rms})+/$-600(inst.) \\
\hline 8.- Power (Note 1) & 160 watts \\
\hline 9.- SNR out & $>63 \mathrm{~dB}$ \\
\hline
\end{tabular}

A characteristic that specially has been studied has been the harmonic distortion of the amplifier. In order to guarantee the global operation of the equipment, it has been necessary to carry out measures of this parameter at different input frequencies and different power outputs.

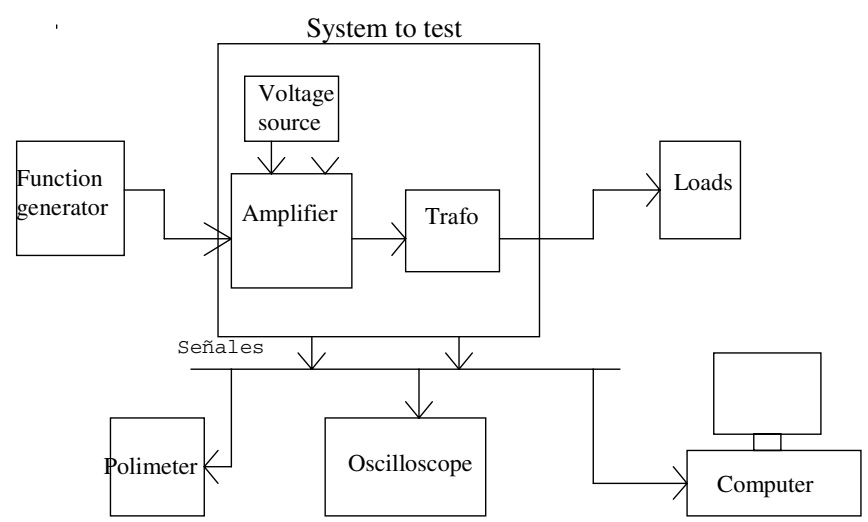

Fig. 12. Scheme of the assembly to test the amplifier

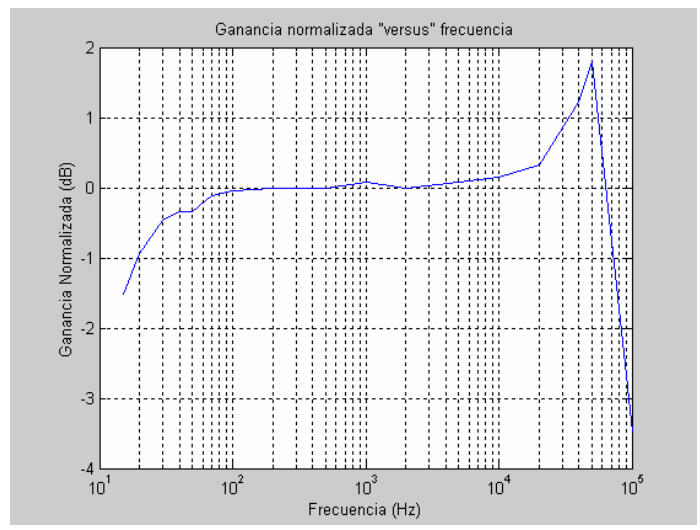

Fig. 13. Gain 'versus' frequency (BW) 
We developed a low cost experimental tool, based on PC with standard audio board and MATLABC, for the measurement of Harmonic Distortion.
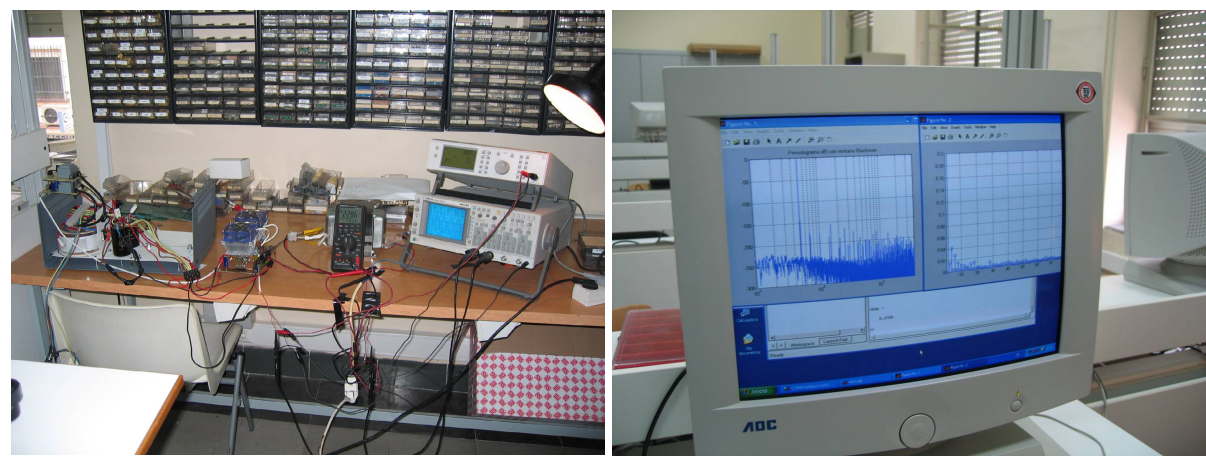

Fig. 14. Experimental setup and Harmonic Distortion Measurement System

This tool, based on DFT, allows us to measure for different inputs frequencies and output powers the $n$ order Harmonic Distortion Index HDi, and the Total Harmonic Distortion (THD). The THD of the amplifier fulfilled the requirements (THD $<0.2 \%$ ) for all the bandwidth $(25 \mathrm{~Hz}-5 \mathrm{Khz})$ and for powers up to 160 watts.
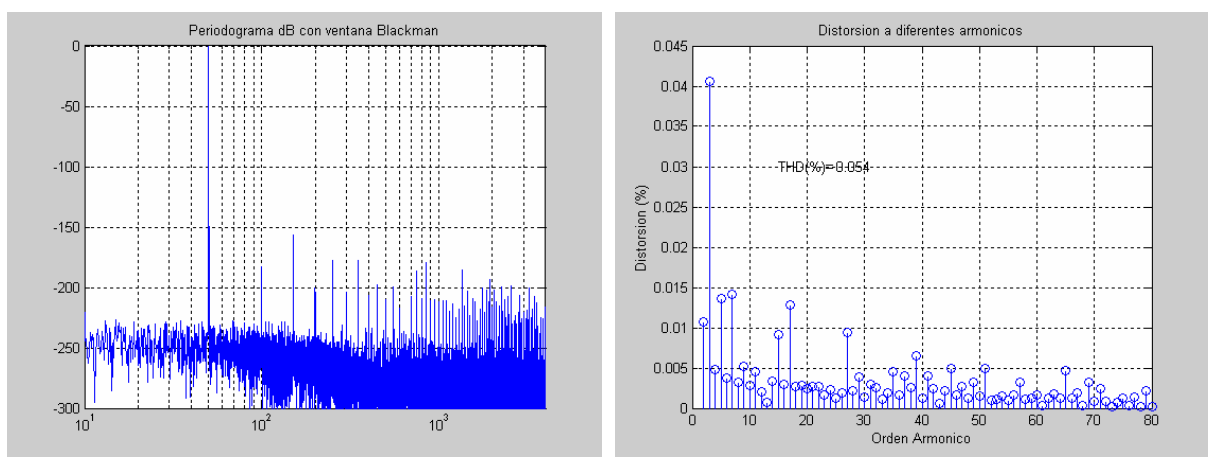

Fig. 15. Periodogram of output signal (Input $0 \mathrm{~Hz}$ ) and Measurement of HDi index and THD

\section{Conclusions}

Today it is known that neural networks are a good choice for detecting and classifying electrical power disturbances. Often the problem lies in generating a sufficient number of training patterns to get that neural networks obtain good results in future inputs.

We have developed an electrical pattern generator which is capable of generating common disturbances which can be found in a power line with the aim of making the training of neural networks easier. The tool can generate a set of disturbance data which include: impulse, oscillation, sag, swell, interruption, undervoltage, overvoltage, harmonics, flicker and frequency variations. In addition, it can add different 
kinds of noise to the generated signals. This generator has been recently patented by the University of Seville.

We have added a high fidelity power amplifier specifically developed for the application that allows obtained outputs at real level in terms of voltage and with small power levels (up to 200 watts). This paper describes the generator, the different mathematical models for each disturbance as well as the power amplifier and the global performance of the system.

\section{Acknowledgments}

The work described in this paper has been supported by the Spanish Ministry of Science and Technology (MCYT: Ministerio de Ciencia y Tecnología) through project reference number DPI2006-15467-C02-02.

\section{References}

[1] McGranaghan, M., Roettger, B.: Economic Evaluation of Power Quality. 0272-1724/02 (2002)

[2] Hussain, A., Sukairi, M.H., Mohamed, A., Mohamed, R.: Automatic Detection Of Power Quality Disturbances and Identification of Transients Signals. In: International Symposium on Signal Processing and its Applications, Kuala Lumpur, Malaysia, August 13-16, 2001 (2001)

[3] Adapa, R.: Power Quality Analysis Software, 0272-1724/02 IEEE (2002)

[4] Anis Ibrahim, W.R., Morcos, M.M.: Artificial Intelligence and Advanced Mathematical Tools for Power Quality Applications: A Survey. 668 IEEE Transactions on Power Delivery 17 (2002)

[5] Zheng, G., Shi, M.X., Liu, D., Yao, J., Mao, Z.M.: Power Quality Disturbance Classification Based on Rule-Based and Wavelet-Multi-Resolution Decomposition. In: Proceedings of the First International Conference on Machine Learning and Cybernetics, Beijing, November 4-5 (2002)

[6] Lai, L.L.: Wavelet-based Neural Network for Power Quality Recognition. 0-7803-73227, IEEE (2002)

[7] Xiangxun, C.: Wavelet-based Measurement and Classification of Power Quality Disturbances, 0-7803-7242-05/02, IEEE (2002)

[8] Borrás, D., Castilla, M., Moreno, N., Montaño, J.C.: Wavelet and neural structure: a new tool for diagnostic of power system disturbances. IEEE Trans. on Industry Applications 37(1), 184-190 (2001)

[9] Bhargava, B.: Arc Furnace Flicker Measurements and Control. IEEE Transactions on Power Delivery 8(1), 409-423 (1993)

[10] Topalis, F.V., Gonos, I.F., Vokas, G.A.: Arbitrary wavwform generator for harmonic distortion test on compact fluorescent lamps. Measurement 30, 257-267 (2001)

[11] Anderson, L.M., Bowes, K.B.: The effects of Power-line disturbances on consumer electronic equipment. IEEE Transactions on Power Delivery 5(2), 1062-1065 (1990) 